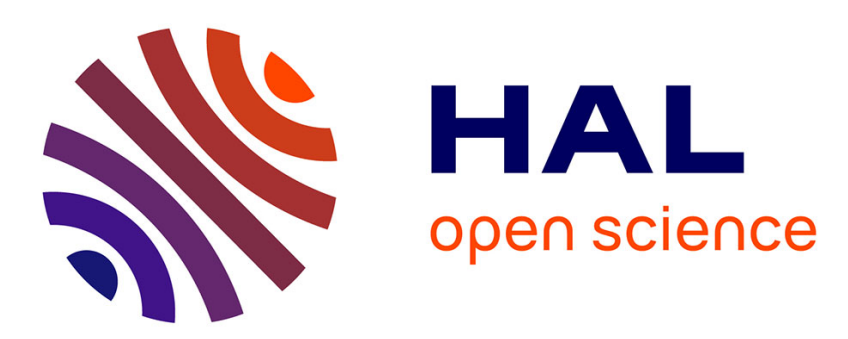

\title{
Appareillage de mesure des chaleurs spécifiques à basse température
}

\author{
J. Bonnerot
}

\section{To cite this version:}

J. Bonnerot. Appareillage de mesure des chaleurs spécifiques à basse température. Revue de Physique Appliquée, 1966, 1 (1), pp.61-67. 10.1051/rphysap:019660010106100 . jpa-00242685

\section{HAL Id: jpa-00242685 https://hal.science/jpa-00242685}

Submitted on 1 Jan 1966

HAL is a multi-disciplinary open access archive for the deposit and dissemination of scientific research documents, whether they are published or not. The documents may come from teaching and research institutions in France or abroad, or from public or private research centers.
L'archive ouverte pluridisciplinaire HAL, est destinée au dépôt et à la diffusion de documents scientifiques de niveau recherche, publiés ou non, émanant des établissements d'enseignement et de recherche français ou étrangers, des laboratoires publics ou privés. 


\title{
APPAREILLAGE DE MESURE DES CHALEURS SPÉCIFIQUES A BASSE TEMPÉRATURE
}

\author{
Par J. BONNEROT $\left({ }^{\mathbf{1}}\right)$,
}

Centre de Recherches de la C. G. E., Marcoussis, Faculté des Sciences d'Orsay, Physique des Solides.

\begin{abstract}
Résumé. - On décrit une méthode adiabatique de mesures des chaleurs spécifiques dans un domaine de température compris entre 2 et $40^{\circ} \mathrm{K}$. L'énergie servant à l'échauffement de l'échantillon est délivrée à puissance constante, et la mesure consiste à enregistrer la loi de variation de la température en fonction du temps. Le couplage écran échantillon mesuré à la température la plus basse a pu être évalué à $4,7 \times 10^{-7} \mathrm{w}^{0} \mathrm{~K}^{-1}$ et les performances ont été vérifiées par l'étude d'un échantillon de plomb pur.
\end{abstract}

Abstract. - An adiabatic method of measurement of specific heats is described. The experiments are made between 2 and $40^{\circ} \mathrm{K}$. The energy used for heating is transmitted to the sample at a constant rate of power. We record the variation of temperature as a function of time. The coupling constant measured at the lowest temperature can be evaluated as $4.7 \times 10^{-7} \mathrm{w}^{0} \mathrm{~K}^{-1}$. The properties of the apparatus are verified by studying a pure lead sample.

I. Introduction. - Les méthodes calorimétriques de mesure (méthodes des mélanges, méthode de flux de chaleur, méthode adiabatique) peuvent se résumer par une équation du type :

$$
\Delta Q=\int_{t}^{t+\Delta t} M C \frac{\mathrm{d} T}{\mathrm{~d} t} \mathrm{~d} t+\int_{t}^{t+\Delta t} K \delta T(t) \mathrm{d} t
$$

où $\Delta Q$ est un échange d'énergie de type différent suivant la nature de la méthode, $M C$ la capacité calorifique de l'échantillon et des accessoires, de masse $M$ et de chaleur spécifique $C, K$ le coefficient de couplage thermique entre l'échantillon et l'en. ceinte, et $\delta T$ leur écart de température, enfin $\Delta T$ représente l'élévation de température de l'échantillon pendant le temps $\Delta t$. Le premier terme donne accès aux chaleurs spécifiques, le second représente les échanges avec l'extérieur.

Dans un calorimètre adiabatique tel qu'il a été initialement décrit par Nernst la seconde intégrale est minimisée en introduisant entre l'échantillon et l'ambiance (paroi externe du calorimètre) un écran maintenu constamment à la température de l'échantillon ( $\delta T$ à peu près nul) et découplé thermiquement de celui-ci ( $K$ équivalent de zéro).

La mesure consiste alors à relever l'évolution de la température en fonction de l'énergie dissipée dans l'échantillon. En pratique on dissipe cette énergie à puissance constante $W$ et on enregistre la courbe de la température en fonction du temps $(T=f(t))$.

(1) Cette étude a été entreprise à l'occasion du contrat D. G. R. S. T. no 62.FR.114.
De cette courbe, on peut tirer la chaleur spécifique :

$$
C_{\mathbf{p}} \# C_{\mathrm{v}}=\frac{\mathrm{d} Q}{\mathrm{~d} T} \# \frac{\mathrm{d} H}{\mathrm{~d} T}=\frac{1}{M} \frac{W}{f^{\prime}(t)}
$$

l'enthalpie

$$
\Delta H_{12}=\int_{t_{1}}^{t_{2}} W \mathrm{~d} t=\frac{W}{M}\left(t_{2}-t_{1}\right)
$$

enfin l'entropie

$$
\Delta S_{12}=\int_{1}^{2} \frac{\mathrm{d} \Delta H}{T}=\frac{W}{M} \int^{t_{2}} \frac{\mathrm{d} t}{f(t)} .
$$

Nous avons étudié et réalisé sur ce principe un calorimètre fonctionnant entre 2 et $40^{\circ} \mathrm{K}$.

La méthode choisie apporte une grande simpl:fication d'abord au point de vue rapidité et facilité d'enregistrement de la mesure et ensuite au point de vue dépouillement des données expéiimental:s et utilisation des renseignements donnés par les mesures comme le montrent les équations ci-dessus.

II. Description et technique expérimentale. Les mesures se font en deux étapes, d'abord de $4,2 \mathrm{~K}$ à $40^{\circ} \mathrm{K}$, puis de la température minima à 5 ou 6 o K. L'échantillon est amené à la température de début d'expérience au moyen de gaz d'échange (la mise en équilibre thermique par contact mécanique présentant à ce stade de trop grandes difficultési. Après pompage de ce gaz, l'échantillon se trouve isolé thermiquement et la fourniture d'une énergie à puissance constante provoque l'élévation 
de température à enregistrer. Une source indépendante de celle utilisée pour le chauffage de l'échantillon alimente l'écran de façon à la maintenir à la même température que l'échantillon ' $(\delta T \sim 0)$. Les données recueillies sont ensuite dépouillées à l'aide d'une machine I. B. M. 7094.

Nous décrirons successivement la partie cryogénique et mécanique, puis la partie électrique.

1. Cryostat et calorimètre. - Le couplage thermique peut se faire par rayonnement, conduction et convection, facteurs qui conditionnent l'autonomie du cryostat. Le rayonnement est minimisé en polissant de façon poussée deux surfaces en regard portées à des températures différentes. Les pertes par conduction sont occasionnées par l'existence des parois métalliques et des fils de connexion. Les parois seront en acier inoxydable mince (de l'ordre de 5/10), les fils de connexion électrique en constantan de $5 / 100 \mathrm{~mm}$ de diamètre, et les fils de suspension de l'échantillon en nylon de $8 / 100 \mathrm{~mm}$ de diamètre. La convection est éliminée par pompage du gaz d'échange introduit pour la mise en température de l'échantillon et nous constatons que, à

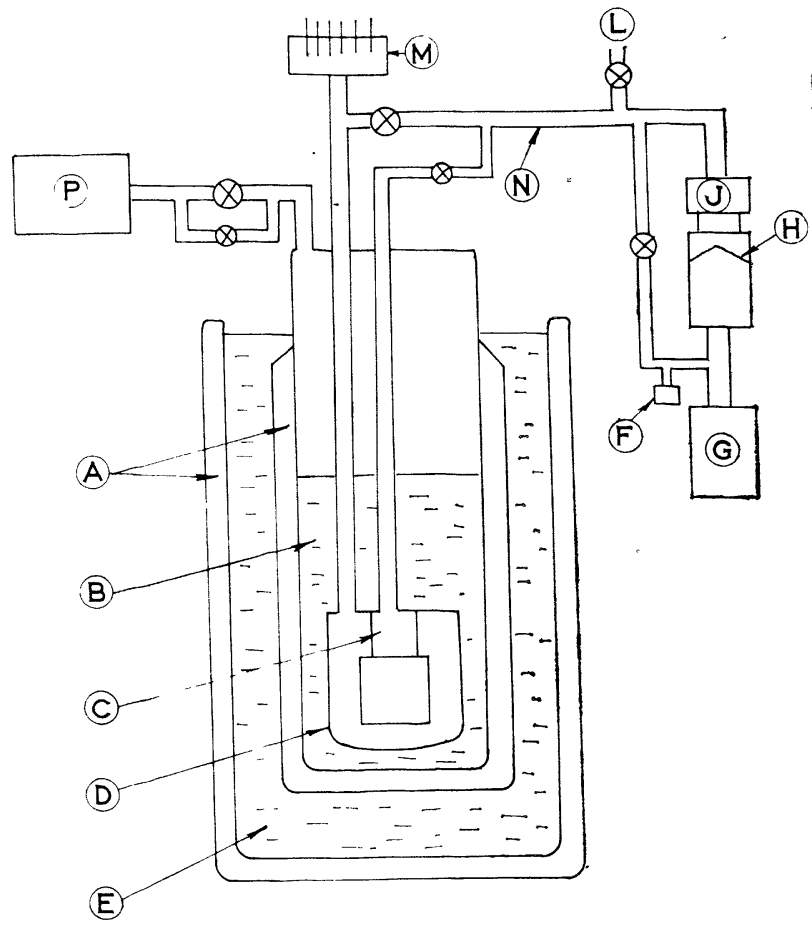

FIg. 1. - Schéma du dispositif de pompage sur le cryostat. A : Vide. - B : Hélium liquide. C : Échangeur. - D : Calorimètre. - E : Azote liquide. - F: Entrée d'air. - G : Pompe primaire. - $\mathrm{H}$ : Pompe secondaire. - $\mathrm{J}$ : Piège à $\mathrm{N}_{2}$ liquide. - L : Entrée d'He gazeux (gaz d'échange). $\mathrm{M}$ : Connexions électriques étanches. - N : Pompage du gaz d'échange. - P : Pompe primaire pour pompage sur bain d'hélium. - Point R: Limite supérieure de la garde de vide du vase hélium. $4,2^{\circ} \mathrm{K}$, pour avoir des pertes inférieures à $10^{-7}$ watt, il faut mesurer à la température ambiante une pression inférieure à $5 \times 10^{-6}$ Torr.

a) Cryostat. - Il est schématiquement représenté sur la figure 1. Il se compose de deux vases métalliques indépendants, munis de garde de vide, destinés à recevoir, l'un l'azote liquide, l'autre l'hélium liquide. La gaine de vide du vase hélium est limitée en hauteur au point $R$ de sorte que en ce point les vapeurs d'hélium soient à la température de $77^{\circ} \mathrm{K}$. Les gradients thermiques dans le cryostat sont ainsi parfaitement définis.

A l'intérieur du cryostat sont disposés trois tubes de pompage, à l'intérieur desquels passent les fils de connexion. Les pertes totales peuvent s'évaluer à $60 \mathrm{~mW}$ ce qui, pour un remplissage de 2,5 litres, assure une autonomie théorique de $24 \mathrm{~h}$ et pratique de 30 heures.

Enfin pour descendre à la température minima, le cryostat est relié à une pompe primaire de $25 \mathrm{~m}^{3} / \mathrm{h}$ de débit. La température limite de $1,5{ }^{\circ} \mathrm{K}$ est atteinte au bout d'une heure et demi de pompage.

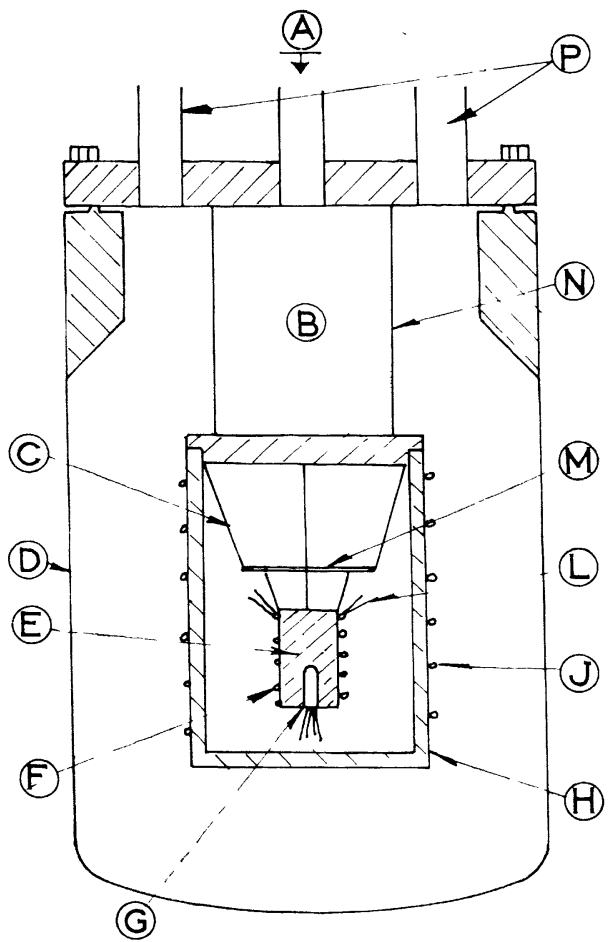

FIG. 2. - Schéma du calorimètre. - A : Pompage sur l'échangeur. - B : Réservoir d'He utilisé comme source de froid pour la régulation. -- $\mathrm{C}$ : Fil de nylon $8 / 100 l=100 \mathrm{~mm}$. - D : Enceinte externe. E : Échantillon. - F : Chauffage échantillon. $\mathrm{G}$ : Thermomètre germanium. - $\mathrm{H}$ : Écran en cuivre. - J : Enroulement chauffant de l'écran. - L : Fils électriques constantan $5 / 100, l=100 \mathrm{~mm}$. M : Plaque relais acier inoxydable ép. $5 / 10 \mathrm{~mm}$. $\mathrm{N}$ : Tôle d'acier inoxydable ép. $1 / 10, l=50 \mathrm{~mm}$. $\mathrm{P}$ : Pompage sur le calorimètre et passage des fils électriques. 
b) Calorimètre. - Il est schématisé sur la figure 2. C'est une enceinte étanche susceptible d'être amenée sous vide secondaire (10-6 Torr). L'échantillon disposé au centre et percé d'un trou pour le logement du thermomètre est suspendu à l'écran au moyen de trois fils de nylon. La mise en température est assurée par gaz d'échange, la réalisation d'un contact mécanique s'est révélée trop délicate, dangereuse pour la suspension. Le gaz d'échange utilisé peut être soit de l'hélium 4 soit de l'hélium 3 qui possède une tension de vapeur supérieure et ne présente pas de transition suprafluide à $2,18^{\circ} \mathrm{K}$. Le pompage du gaz est assuré par une pompe primaire de $25 \mathrm{~m}^{3} / \mathrm{h}$, une pompe secondaire de $50 \mathrm{l} / \mathrm{sec}$ à $10^{-4}$ Torr, surmontée d'un piège à azote liquide ( $f i g .1)$.

Entre l'échantillon et la bride d'écran est disposé un écran flottant en acier inxoxydable qui sert de relais pour les fils électriques.

La partie supérieure de l'écran support de l'échantillon est en cuivre. La partie mobile de l'écran (cylindre de cuivre) y est fixée mécaniquement et reliée thermiquement au moyen du vernis G. E. 7031 bon conducteur de la chaleur.

L'écran est relié à l'enceinte externe du calorimètre par l'échangeur, cylindre en acier inoxydable de $40 \mathrm{~mm}$ de diamètre, $50 \mathrm{~mm}$ de longueur et de 1/10 mm d'épaisseur de paroi, sans communication avec l'intérieur du calorimètre. Le calorimètre luimême se compose d'une enceinte externe isotherme en cuivre qui baigne dans l'hélium liquide. Il est constitué d'une partie fixe d'où partent les tubes nécessaires au pompage et au passage des connexions électriques, et d'une partie mobile. L'étanchéité entre les deux est assurée au moyen d'un joint téflon de $2 / 10 \mathrm{~mm}$ d'épaisseur serré sur la périphérie de la bride.

2. Dispositif de mesure. - L'ensemble est schématisé sur la figure 3 . Il se subdivise en trois grandes parties indépendantes: le chauffage de l'échantillon, la régulation d'écran et la mesure de température.

Chauffage de l'échantillon. - Pour les mesures en continu, la puissance délivrée à l'échantillon doit être stabilisée à $\pm 1 \%$ dans l'intervalle de température considéré. Or la valeur de la résistance chauffante varie en fonction de la température : il sera en conséquence nécessaire de scinder l'intervalle $2-40^{\circ} \mathrm{K}$ en plusieurs domaines. La variation de puissance restera inférieure à la limite fixée si la valeur de la résistance chauffante reste comprise entre 80 et $120 \%$ de celle du générateur. Il est facile de vérifier en cours de manipulation que ces conditions sont respectées. La puissance V. I. est déterminée par mesures potentiométriques (le courant est mesuré aux bornes d'une résistance étalon de $100 \Omega$ définie à $10^{-5}$ près). Les prises de potentiel aux bornes de l'élément chauffant se font suivant une méthode préconisée par Hoare et reprise par

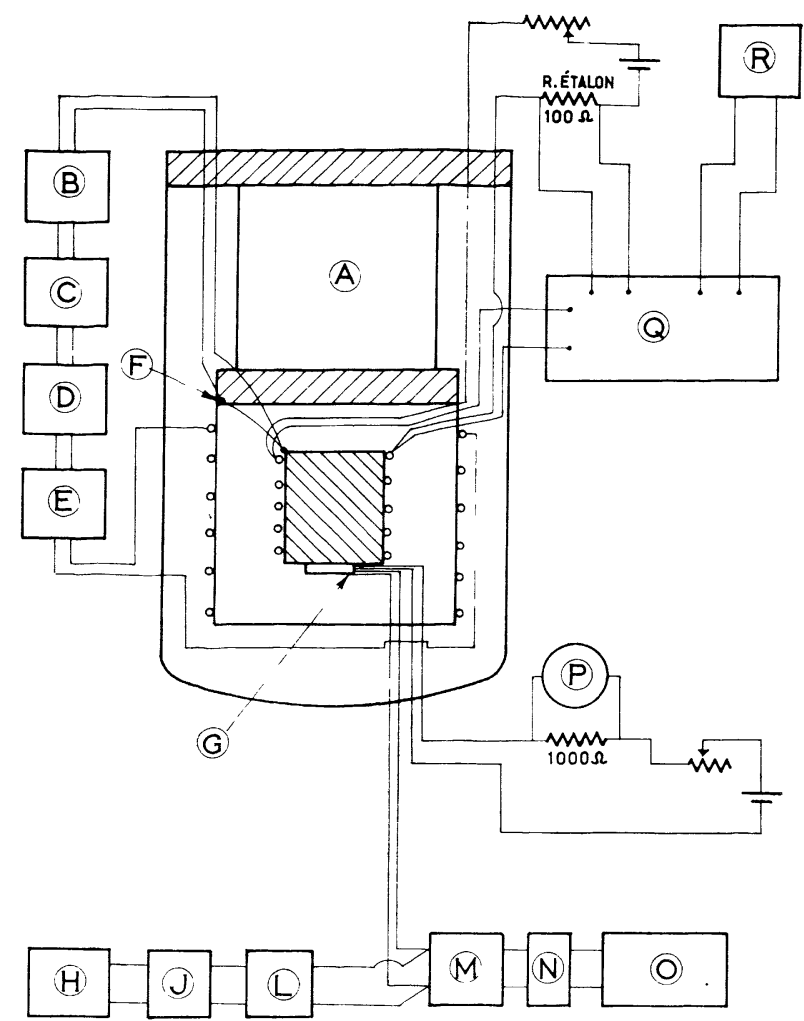

FIG. 3. - Schéma du dispositif électrique de mesures de chaleurs spécifiques. A : Échangeur. - B : Amplificateur continu Guildine $\mathrm{G}=4000$. - C : Speedomax Méci. — D : Amplificateur Leeds-Northrup. E : Amplificateur C. G. E. sortie : $100 \mathrm{~V}$ sur $2000 \Omega$. - F : Thermocouple Au 2,1\% Co Normal Silver. $\mathrm{G}$ : Thermomètre. - $\mathrm{H}$ : Suiveur de sport. - J : Galvanomètre SEFRAM. - L: Amplificateur différentiel. - $\mathrm{M}$ : Voltmètre digital. - $\mathrm{N}$ : Transcodeur. - $\mathrm{O}$ : Perforatrice I. B. M. - P : Galvanomètre SEFRAM. $\mathrm{Q}$ : Pont Cambridge Microstep. - R : Galvanomètre Kipp A15.

Conte. La puissance dépensée pour élever la température de l'échantillon est de la forme :

$$
W=r I^{2}+\left(\left(r_{1}+r_{2}\right) / 2\right) I^{2}
$$

où $r$ désigne la résistance de l'élément chauffant, $r_{1}$ et $r_{2}$ les résistances des fils de jonction entre l'écran et le bobinage. L'expression ci-dessus est un moyen terme entre $\mathfrak{w}=r I^{2}$ puissance dissipée dans l'échantillon et $w=r I^{2}+\left(r_{1}+r_{2}\right) I^{2}$ puissance dissipée dans le calorimètre. Les valeurs calculées avec la première formule sont valables si $2 r /\left(r_{1}+r_{2}\right)$ est supérieure ou égal à 50 , ce qui est le cas présent [1].

Régulation d'écran. - L'égalité des températures entre écran et échantillon est assurée par une régulation asservie commandée par un thermocouple différentiel or $2,1 \%$ cobalt et argent-or. Le pouvoir thermoélectrique varie de $2,07 \mu \mathrm{V} /{ }^{\circ} \mathrm{K}$ à $2{ }^{\circ} \mathrm{K}$ à $26,23 \mu \mathrm{V} /{ }^{\circ} \mathrm{K}$ à $40^{\circ} \mathrm{K}[2]$. Les branches de descente 
sont constituées par de l'argent-or, et la longueur de fil or-cobalt doit être rendue minimum (compatible cependant avec l'élimination des pertes par conduction) car ce matériau est soumis à des variations de concentration. Quand les fils (5/100 de $\mathrm{mm}$ de diamètre) sont soudés il est indispensable de s'assurer que les pouvoirs thermoélectriques des deux soudures sont identiques dans toute la gamme de température. Il faut d'ailleurs recommencer cette vérification de temps en temps pour s'assurer de la fidélité. (Le phénomène est surtout important aux basses températures où les sensibilités deviennent faibles).

On s'impose qu'entre écran et échantillon l'écart de température n'excède jamais $10^{-2} \mathrm{oK}$ ce qui aux plus basses températures représente $2 \times 10^{-2} \mu \mathrm{V}$. Ce signal amplifié commande un régulateur qui se déclenche pour des signaux supérieurs ou égaux à $50 \mu \mathrm{V}$. Pour avoir le gain nécessaire (i. e. supérieur à 2500 ) nous avons utilisé un amplificateur Guildline de $3 \times 10^{-2} \mu \mathrm{V}$ pleine échelle à l'entrée et de très faible dérive $\left(5 \times 10^{-3} \mu \mathrm{V}\right.$ en 24 heures $)$. La boucle d'asservissement comporte en série à la sortie de cet amplificateur un potentiomètre enregistreur Méci, un régulateur Leeds et Northrup, enfin un amplificateur continu susceptible d'alimenter sous 100 volts une résistance chauffante de $2000 \Omega$ environ bobinée autour de l'écran.

Elle constitue la source de chaleur nécessaire pour que l'écran se réchauffe en même temps que l'échantillon. Il faut lui adjoindre une source froide (fig. 2). De l'hélium introduit sous basse pression (environ $10^{-3}$ Torr) dans l'échangeur amène par convection de frigories de la partie supérieure du calorimètre à la bride supérieure de l'écran.

Mesure de la température. - Le thermomètre est l'autre élément essentiel de la mesure. Il doit être fidèle, sensible, reproductible. Les trois qualités ne sont pas remplies par une résistance de carbone surtout au-dessus de $8^{\circ} \mathrm{K}$. Le thermomètre germanium répond aux conditions fixées. Il a été fourni par Honeywell qui s'est d'ailleurs chargé de l'étalonnage, et possède une reproductibilité supérieure à 10-4. La résistance varie de façon monotone de $15000 \Omega$ à $1,5 \circ \mathrm{K}$ à $14 \Omega$ à $40^{\circ} \mathrm{K}$. La puissance dissipée dans la sonde ne doit pas excéder $3 \mu \mathrm{W}$ ce qui conditionne les valeurs des courants d'utilisation repérés aux bornes d'une résistance étalon de $1000 \Omega$ définis à $10^{-4}$ près.

La tension aux bornes du thermomètre est envoyée d'une part aux bornes d'un voltmètre digital Dymec $2401 \mathrm{~B}$, de sensibilité $1 \mu \mathrm{V}$ sur le dernier digit, couplé par l'intermédiaire d'un transcodeur à une perforatrice de cartes, et d'autre part à travers un amplificateur différentiel à un galvanomètre solidaire d'un suiveur de spot. Le voltmètre digital permet une lecture absolue alors que le suiveur de spot permet de contrôler le déroulement de la mesure de façon qualitative car l'amplificateur différentiel n'a pas un gain parfaitement égal à un et possède de plus une certaine dérive.

3. Appréciation des performances. - Le système de pompage utilisé permet d'obtenir un vide de l'ordre de 10-6 Torr. Les pertes par convection, compte tenu des surfaces en regard peuvent être évaluées à $0,3 \times 10^{-7}$ watt. Pour les fils de connexions électriques et les fils de thermocouple, les pertes peuvent être calculées à partir des coefficients moyens de conductibilité thermique. Elles sont igales en watt à $1,5 \times 10^{-7} \Delta T$ où $\Delta T$ est l'écart de température entre écran et échantillon. Celles provoquées par les fils de nylon sont au moins dix fois plus faibles que les autres : elles peuvent donc être négligées ainsi que les pertes par rayonnement (équivalentes à 10-9 watt).

Les pertes estimées vont rester constantes tout au long de l'expérience car le rapport des coefficients de conduction thermique à la sensibilité du thermocouple reste à peu près constant en fonction de la température. Le premier facteur conditionne le terme $1,5 \times 10^{-7}$ et le second la valeur de $\Delta T$.

De façon à mettre en évidence le découplage entre échantillon et enceinte, l'ensemble est amené à la température la plus basse, puis l'échantillon isolé de l'écran par pompage du gaz d'échange. L'échantillon commence à s'échauffer; en effet en dessous de $4,2{ }^{\circ} \mathrm{K}$ les vibrations, provoquées par les pompes primaires, et partiellement transmises à l'échantillon. provoquent un échauffement. Au-dessus de 4,2 o K cet effet devient totalement négligeable. L'énergie apportée par les vibrations peut s'évaluer à $2 \times 10^{-7} \mathrm{~W}$ et l'énergie apportée par le thermomètre de résistance $10^{4} \Omega$ à $2{ }^{\circ} \mathrm{K}$ et alimenté par un courant de $7 \mu \mathrm{A}$ est de $5 \times 10^{-7} \mathrm{~W}$. Dans ces conditions un échantillon de plomb pur pesant environ $30 \mathrm{~g}$, de chaleur spécifique à $2{ }^{\circ} \mathrm{K}$ égale à $9 \times 10^{-2} \mathrm{~mJ} / \mathrm{g}$ oK s'est trouvé porté au bout d'une heure à une tempéragure de $0,7^{\circ} \mathrm{K}$ supérieure à celle de l'écran, ce qui signifie un coefficient moyen de couplage de $\iota_{4} \times 10^{-7} \mathrm{Watt} \mathrm{deg}^{-1}$ (la puissance typique utilisée en cours de mesure est de l'ordre de $10^{-5}$ watt).

La première intégrale de (I) fait intervenir le produit $M C$ somme des contributions de l'échantillon et des accessoires. Les accessoires comprennent le fil de bobinage, le vernis qui le maintient fixé sur l'échantillon, et le thermomètre. A $4^{\circ} \mathrm{K}$ par exemple, les deux premiers interviennent pour $35 \mu \mathrm{J} /{ }^{\circ} \mathrm{K}$, le thermomètre a une capacité calorifique due au cuivre de la capsule du thermomètre au germanium, élément détecteur, qui peut être évaluée à $80 \mu \mathrm{J} /{ }^{\circ} \mathrm{K}$, soit au total $115 \mu \mathrm{J} /{ }^{\circ} \mathrm{K}$. Avec un échantillon de $30 \mathrm{~g}$, la capacité calorifique des accessoires est inférieure à $1 / 100$ de celle de l'échantillon. De plus, cette erreur est du même ordre de grandeur et de sens opposé à celle commise en négligeant la quantité de chaleur apportée par la sonde de mesure. 
Nous pourrons donc négliger l'ensemble de ces corrections pour ces deux effets.

Dans ces conditions, l'erreur expérimentale totale due aux erreurs de mesure, aux erreurs systématiques, et à la dispersion expérimentale inévitable peut être évaluée à $2,5 \%$.

III. Mesures de la chaleur spécifique du plomb. Pour contrôler les performances de l'appareil nous avons déterminé la chaleur spécifique du plomb pur. L'échantillon étudié de forme cylindrique allongée, pesait $31,327 \mathrm{~g}$ et nous avait été fourni par Hoboken avec un titre nominal de 99,999\%. Son analyse par voie chimique n'a laissé apparaître aucune trace de matériaux ferromagnétiques comme le fer ou le nickel susceptibles d'être responsables d'un abaissement de la température de transition supraconductrice.

Les mesures ont été réalisées à la fois en continu et par passage d'impulsion de courant. L'adiabatisme a été contrôlé dans les deux cas et nous avons pu constater que la mise en température de l'échantillon n'excédait jamais 30 secondes.

Lors des mesures à puissance constante, la puissance délivrée à l'échantillon passe de $15 \mu \mathrm{W}$ à $2{ }^{\circ} \mathrm{K}$ à $15 \mathrm{~mW}$ à $400^{\circ} \mathrm{K}$; les valeurs de la tension aux bornes du thermomètre sont relevées toutes les 30 ou les 60 secondes suivant le domaine de température.

Entre 30 et $40^{\circ} \mathrm{K}$ afin de minimiser $\int_{t_{1}}^{t_{2}} K \delta T \mathrm{~d} t$ lors de mesures de chaleur spécifique, par impulsion de courant, il faut envoyer à l'échantillon une puissance assez faible; en effet, l'application brutale d'une forte puissance déséquilibre la régulation et fait apparaître un $\delta T$ qui peut être de l'ordre de $1 / 10^{\circ} \mathrm{K}, \delta T$ qui redevient nul au bout d'une minute environ. Aussi pour avoir des échauffements de l'échantillon qui permettent d'éliminer les causes d'erreur, il sera nécessaire de lui fournir une énergie moindre mais pendant un temps plus long (au minimum de l'ordre de 2 minutes). Aux basses températures cet inconvénient ne se présente pas et la durée des mesures peut être de 30 secondes.

Les résultats de la mesure de la chaleur spécifique du plomb pur (en l'absence de champ magnétique) sont représentés sur la figure 4. La transition supraconductrice apparaît plus nettement sur le tracé de $C / T$ en fonction de $T^{2}$; elle se produit à $7,19{ }^{\circ} \mathrm{K}$, ce qui correspond à la température de transition théorique. Les valeurs de comparaison ont été établies par Horowitz, Silvidi, Malaker et Daunt en 1952 et reprises par les tables du N. B. S. [3] (tableau I). La comparaison se révèle bonne, l'erreur maxima ayant lieu autour de $6 \circ \mathrm{K}$ où elle est de $6 \%$. A $40 \% \mathrm{~K}$ nous avons pu constater que l'adiabatisme était excellent puisque la stabilisation en température de l'échantillon s'est produite 30 secondes après avoir cessé de lui fournir de l'énergie, ce qui représente

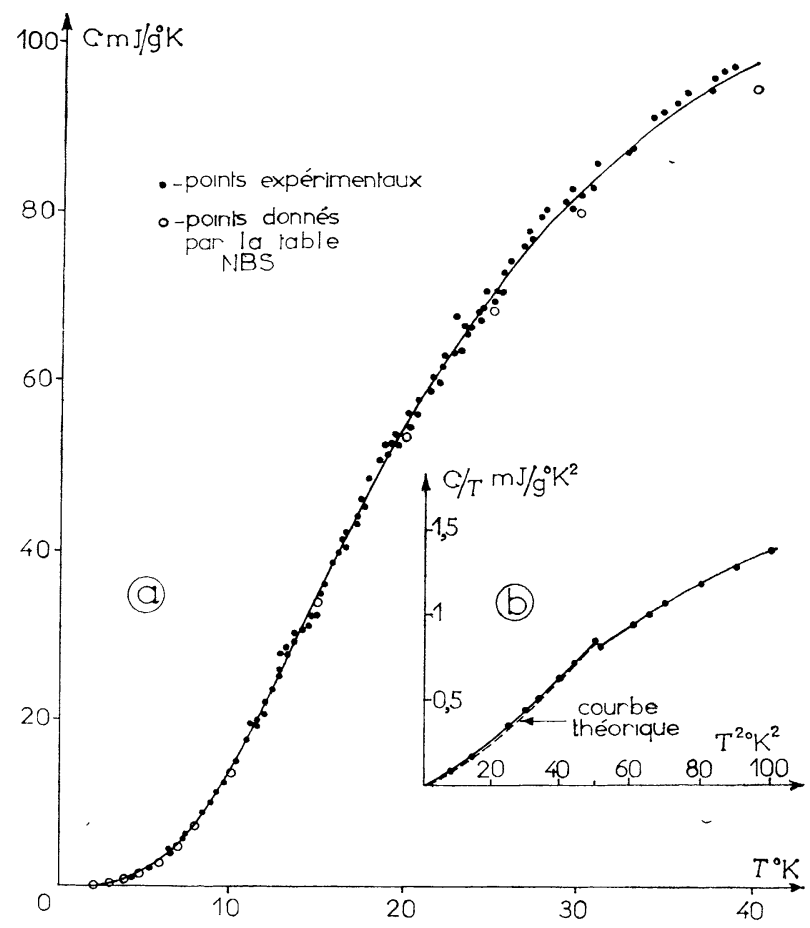

Frg. 4. - A : Variation de la chaleur spécifique du plomb en fonction de la température. Plomb Hoboken $99,999 \%$ - B : Variation du rapport $C / T$ en fonction de $T^{\mathbf{2}}$.

une traînée de l'ordre de $0,03{ }^{\circ} \mathrm{K}$. Des résultats identiques ont été obtenus entre 4 et $5{ }^{\circ} \mathrm{K}$ avec le même échantillon chauffé avec une puissance de $36 \mu \mathrm{W}$ pendant des intervalles de temps égaux à 30 , $60,90,120$ secondes (tableau II).

Keesom et Van der Hoeven Jr [4] ont mesuré la chaleur spécifique du plomb entre 0,4 et $4,2{ }^{\circ} \mathrm{K}$ à l'état normal et à l'état supraconducteur. Leurs résultats sont donnés en fonction de la température par les relations :

$C_{\mathrm{n}}=2,98 T+1,60 T^{3}+0,028 T^{5} \mathrm{~mJ} / \mathrm{mole}^{\circ} \mathrm{K}$

$C_{\mathrm{S}}=0,144 T^{3}+1,60 T^{3}+0,028 T^{5} \mathrm{~mJ} / \mathrm{mole}^{\circ} \mathrm{K}$

où $C_{\mathrm{n}}$ et $C_{\mathrm{S}}$ sont respectivement les chaleurs spécifiques à l'état normal et à l'état supraconducteur.

Nos expériences sont faites en champ nul. Donc en dessous de la température de transition du plomb, nous mesurons seulement $C_{\mathrm{s}}$.

Nos résultats sont bien décrits par la formule :

$$
C_{\mathrm{s}}=1,77 T^{3}+0,035 T^{5} \mathrm{~mJ} / \text { mole }^{\circ} \mathrm{K}
$$

qui est assez voisine de celle de Keesom.

On peut tenter de séparer dans $C_{\mathrm{S}}$ une contribution de réseau $\left(\sim T^{3}\right)$ et une contribution électronique [5]. Admettons que le terme de réseau $C_{\mathrm{r}}$ est le même dans la phase supraconductrice et dans 
TABLEAU I

\begin{tabular}{|c|c|c|c|c|}
\hline \multirow{3}{*}{$T \circ \mathrm{K}$} & \multicolumn{4}{|c|}{$\begin{array}{c}\text { Chaleurs SPÉcifiques } \\
\text { ET ENTHALPIES DU PlOMB MESURÉEs } \\
\text { PAR LA MÉTHOdE CONTINUE }\end{array}$} \\
\hline & \multicolumn{2}{|c|}{ N. B. S. } & \multicolumn{2}{|c|}{ EXPÉRIENCE } \\
\hline & $\begin{array}{c}C p \\
\mathrm{mj} / \mathrm{gr}\end{array}$ & $\underset{\mathrm{mj}}{H}-\underset{\mathrm{g}^{-1}}{H_{0}}$ & $\underset{\mathrm{mj} / \mathrm{gr} \text { oK }}{C p}$ & $\begin{array}{l}H-H_{0} \\
\mathrm{mj} \mathrm{gr}-1\end{array}$ \\
\hline$\overline{1}$ & 0,012 & 0,013 & - & - \\
\hline 2 & 0,09 & 0,05 & $0,12 \quad(2)$ & - \\
\hline 3 & 0,31 & 0,23 & 0,31 & 0,23 \\
\hline 4 & 0,7 & 0,70 & 0,71 & 0,75 \\
\hline 5 & 1,5 & 1,8 & 1,56 & 1,9 \\
\hline 6 & 3,0 & 4,0 & 3,20 & 4,4 \\
\hline 7 & 5,0 & 8,0 & 5,30 & 9 \\
\hline 8 & 7,3 & 14 & 7,80 & 16 \\
\hline 10 & 13,7 & 34 & 13,7 & 36 \\
\hline 15 & 33,5 & 150 & 33,4 & 155 \\
\hline 20 & 53,1 & 368 & 54,3 & 378 \\
\hline 25 & 68,1 & 672 & 68,3 & 692 \\
\hline 30 & 79,6 & 1042 & 80,7 & 1100 \\
\hline 40 & 94,4 & 1920 & 97,2 & 2100 \\
\hline
\end{tabular}

TABLEAU II

Chaleurs spécifiques du plomb OBTENUES PAR CHAUfFAgE PAR IMPULSIONS

$\begin{array}{ccccc}T{ }^{\circ} \mathrm{K} & 4,71 & 4,79 & 4,86 & 4,89 \\ C_{\mathrm{p}} \overline{\mathrm{mJ}} / \mathrm{g}{ }^{\circ} \mathrm{K} & \overline{1,41} & \overline{1,44} & \overline{1,495} & \overline{1,51}\end{array}$

la phase normale. Or d'après les résultats de Keesom, nous prendrons :

$$
C_{\mathrm{r}}=1,60 T^{3}+0,035 T^{5} .
$$

La partie électronique de la chaleur spécifique serait alors, dans la phase supraconductrice

$$
C_{\text {es }}=0,17 T^{3}
$$

mais ce résultat est imprécis, car $C_{\text {es }}$ est une faible fraction de $C_{\mathrm{s}}$. Il faut noter les faits suivants :

1) Le terme de chaleur spécifique électronique est convenablement représenté par une loi en $T^{3}$ alors que suivant la théorie B. C. S. [6] on devrait s'attendre à une loi exponentielle.

$$
C_{\text {es }} / \gamma T_{\mathrm{c}}=8,5 \exp \left(-1,44 T_{\mathrm{c}} / T\right) \text {. }
$$

Toutefois il ne faut pas perdre de vue que les courbes sont numériquement peu différentes dans la région étudiée ( $f g .5)$.

2) Au point critique, le rapport $C_{\mathrm{es}} / \gamma T_{\mathrm{c}}$ vaut 2,62

(2) La valeur à $2{ }^{\circ} \mathrm{K}$ est trop forté à cause du pic de chaleur spécifique de l'hélium 4.

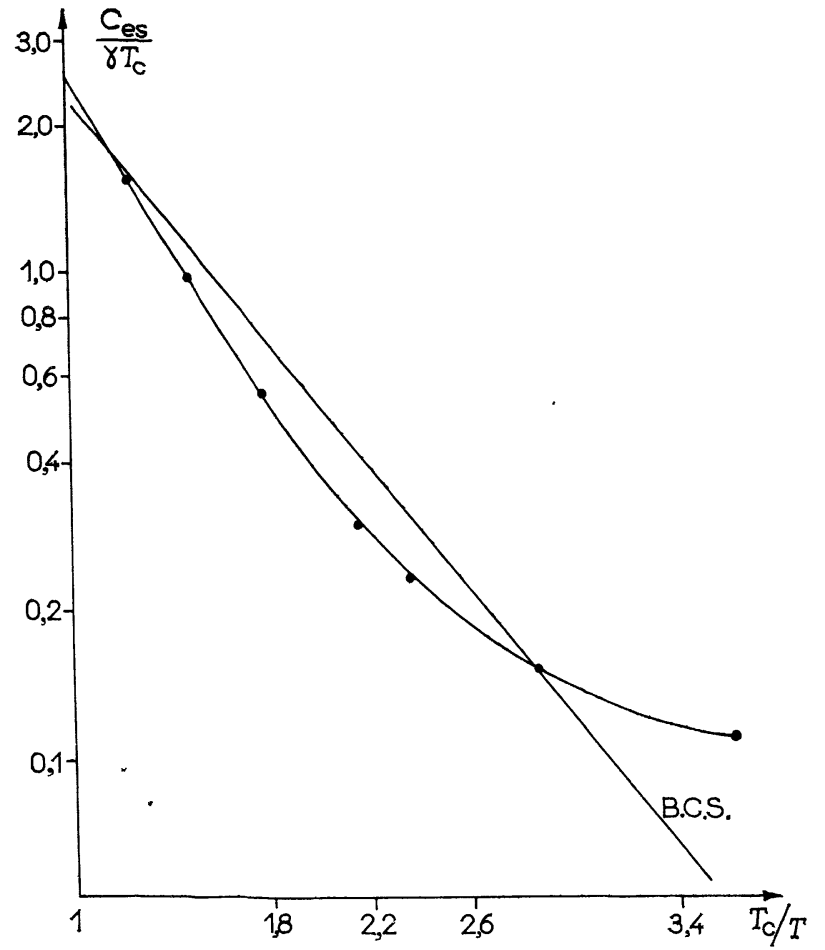

Fig. 5. $-C_{\mathrm{es}} / \gamma T_{\mathrm{c}}$ en fonction de $T_{\mathrm{c}} / T$ pour du plomb.

à comparer avec la valeur 2,43 déduite également de B. C. S. Cet écart est peut-être lié aux effets du " couplage-fort " qui sont connus pour être importants dans le plomb.

En conclusion, il y a un assez bon accord entre nos mesures de chaleurs spécifiques sur le plomb et les valeurs citées dans la littérature. En particulier, la température de transition supraconductrice, déterminée par l'anomalie de chaleur spécifique, est de $7,19^{\circ} \mathrm{K}$ pour notre spécimen ce qui correspond bien aux valeurs connues pour le métal pur $\left(T=7,20^{\circ} \mathrm{K}\right.$ d'après $\left.[3]\right)\left({ }^{3}\right)$. Ces deux faits permettent de penser que les performances attendues du montage (pour l'adiabatisme et pour la mesure des températures) sont effectivement réalisées.

Nous tenons à remercier tout particulièrement M. Blaise pour l'aide qu'il nous a apportée dans la réalisation et la mise au point de l'appareillage électronique, ainsi que M. Le Fur qui a participé activement à la conception et à la réalisation de l'expérience.

( $\left.{ }^{3}\right)$ Un échantillon de plomb contenant $130 \mathrm{ppm}$ de fer a également été mesuré. Sa température de transition était de $6,23 \circ \mathrm{K}$. Cet abaissement spectaculaire correspond bien aux ordres de grandeur cités dans la littérature. Cependant, nous ne savons pas sous quelle forme était le fer dans ce spécimen. 


\section{BIBLIOGRAPHIE}

[1] Hoare et Conte, Communication personnelle, Université de Leeds, C. E. A., Fontenay (Thèse).

[2] Powell Corrucini, Cryogenics, mars 1961, p. 129142.

[3] Horowitz, Silvidi, Mala ker et Daunt, Phys. Res., $1952,88,1282-1286$.
[4] Keesom et Van der Hoeven, Jr., Phys. Letters, 1963, vol. 3, no 7, 360.

[5] Gorter and Daunt, Progress in Low temperatures Physics, North Holland 202, 1957, vol. I.

[6] Bardeen, Cooper et Schrieffer, Phys. Rev., 1957 , 108, 1175. 